patient. For all CD patients a household control was approached. Healthy volunteers stratified by NOD2 genotype were recruited from the Cambridge Bioresource.

Faecal samples were frozen within $24 \mathrm{~h}$ of collection. DNA was extracted using the FASTDNA Spin Kit for Soil. The V1-3 region of the 16S rRNA gene was amplified and amplicons were sequenced with Illumina MiSeq. Sequence data was processed in Mothur. Calprotectin was measured in all samples by ELISA.

Results 97/107 individuals were included in the primary analysis (40 CD patients [58\% NOD2 mutant], 32 bioresource volunteers [50\% NOD2 mutant], 25 household controls). The minimum reads per sample were 3953, mean 21216.

There was a significant reduction in diversity (inverse Simpson index), Ruminococcaceae including Faecalibacteria and increase in Enterobacteriaceae in samples from CD patients vs. controls (all $p<0.0001$ ). There were no differences in diversity or relative abundance of any bacterial families when stratified by NOD2 status, either within the CD patients or bioresource controls.

Conclusion This study confirms previously identified shifts in gut microbiota in CD patients. However, no significant differences in gut microbiota were seen when analysed by NOD2 status. This may be a reflection of sample size or of studying gut bacteria in stool as opposed to the mucosally-associated compartment. We are presently recruiting additional cases and controls to increase study power for additional analysis.

Disclosure of Interest None Declared.

\section{PWE-083 DISTINGUISHING BETWEEN POSSIBLE MENTAL HEALTH DISORDERS AND PSYCHOLOGICAL DISTRESS BY SCREENING FOR ACCEPTANCE AND ADJUSTMENT ISSUES}

${ }^{1} \mathrm{~N}$ Swart ${ }^{*},{ }^{1} \mathrm{D}$ Wellsted, ${ }^{1} \mathrm{~K}$ Friedli, ${ }^{2} \mathrm{G}$ Wood, ${ }^{3} \mathrm{~T}$ Price, ${ }^{3} \mathrm{~K}$ Lithgo, ${ }^{3} \mathrm{M}$ Johnson. ${ }^{1}$ Centre for Lifespan and Chronic IIIness Research, University of Hertfordshire, Hatfield, UK; ${ }^{2}$ Clinical Health Psychology Service, South Essex Partnership Trust, Bedford, UK; ${ }^{3}$ Gastroenterology, Luton and Dunstable University Hospital, Luton, UK

\subsection{6/gutjnl-2014-307263.343}

Introduction A recent study indicated that $30 \%$ of IBD patients suffer from psychological distress, and that poor acceptance and adjustment is a predictor of distress (Swart et al. 2013). Psychological distress encompasses the symptoms of depression, anxiety, emotional difficulty and poor adjustment, but is not the same as mental illnesses such as Major Depressive Disorder or Generalised Anxiety Disorder. Early indications suggest IBD patients are particularly susceptible to adjustment disorders, which may be the cause of much distress.

Methods As part of a service evaluation, 2400 IBD patients in the Luton and Dunstable catchment area were invited to participate in a web-based psychological and quality of life assessment. The "Acceptance and Action Questionnaire" (AAQ-II) was used for acceptance/adjustment, as well as the "Patient Health Questionnaire" (PHQ-9) for depression, and the "Generalised Anxiety Disorder Questionnaire” (GAD-7) for anxiety.

Results 360 patients completed the assessment (45\% male, mean age $=53 ; \mathrm{SD}=17) .31 \%$ of patients scored highly on the PHQ-9; $27 \%$ of patients scored highly on the GAD-7; and 27\% of patients also scored highly on the AAQ-II. Linear regression, after taking account of relevant clinical factors, showed that poor acceptance was associated with high depression and anxiety. Cross tabulation indicated $20 \%$ of patients showed high depression and poor acceptance, and 11\% of patients showed high depression and good acceptance/adjustment. Anxiety sees a similar picture with $18 \%$ high anxiety and poor acceptance/ adjustment, and $8 \%$ high anxiety and good acceptance/ adjustment.

Conclusion The self-report prevalence rates of depression and anxiety we found are typical of chronic illness poplations, however the cross-tabulation results suggest a more complex picture. While further research is needed, theory suggests those $20 \%$ are struggling with depression which stems from their acceptance and adjustment issues. Similarly, those 11\% with high depression and good acceptance are likely to have a mental health disorder like Major Depressive Disorder - $11 \%$ is a similar prevalence rate found when using diagnostic interviews. These groups of patients would likely benefit most from different treatment paths. Specifically, severe depressive symptoms stemming from acceptance/adjustment issues would likely benefit more from Acceptance and Commitment Therapy than standard procedures for treatment of depression with antidepressants and Cognitive Behavioural Therapy.

\section{REFERENCE}

Swart N, Wellsted D, Lithgo K, Price T, Johnson MW. PWE-110 acceptance and adjustment in a district general cohort of inflammatory bowel disease patients: findings and implications. Gut 2013;62:A175-A176

Disclosure of Interest None Declared.

\section{PWE-084 NATIONWIDE LINKAGE ANALYSIS IN SCOTLAND - HAS MORTALITY FOR HOSPITAL ADMISSION FOR CROHN'S DISEASE CHANGED IN THE 21ST CENTURY?}

${ }^{1}$ NT Ventham*, ${ }^{1}$ NA Kennedy, ${ }^{2}$ A Duffy, ${ }^{2}$ DN Clark, ${ }^{3}$ AM Crowe, ${ }^{4}$ A Knight, ${ }^{5}$ JR Nicholls, 1J Satsangi. 'Molecular Medicine Centre, University of Edinburgh, Edinburgh, UK; ${ }^{2} e$ Data Research and Innovation Service (EDRIS), Information Services Division of NHS Scotland, Edinburgh, UK; ${ }^{3}$ Corvus Ltd, Buxted, UK; ${ }^{4}$ Evicom, Teddington, UK; ${ }^{5}$ St Mark's Hospital, London, UK

\subsection{6/gutjnl-2014-307263.344}

Introduction Data from patients hospitalised with Crohn's disease (CD) between 1998 and 2000 in Scotland indicate higher 3 -year mortality rates than the general population. ${ }^{(1)}$ We now have the opportunity of comparing these data with contemporary mortality data in patients admitted in Scotland between 2007-2009. We hypothesised key alterations in management of $\mathrm{CD}$ over the last decade may have reduced mortality.

Methods The Scottish Morbidity Records and linked datasets were used to assess 3-year crude mortality, standardised mortality ratio (SMR) and multivariate analyses of factors associated with 3-year mortality. The 3-year mortality was determined after four admission types: surgery-elective or emergency; medicalelective or emergency. Age-standardised mortality rates (ASR) were used to compare mortality rates between periods.

Results The number of patients hospitalised for 4 or more days with CD was 1460 [Period 1] to 1565 [Period 2] (15.6 to 14.5 per 100,000 Scottish population per year). There was no change in the crude or adjusted 3-year mortality rate between study periods (crude $9.0 \%$ to $9.1 \%$, adjusted OR $=0.87 \mathrm{CI}$ : $0.65-$ $1.17 ; \mathrm{p}=0.355)$. In subgroup analysis, the adjusted 3 -year mortality increased following elective surgery (OR 13.5, CI: $1.66-$ 109.99) and decreased following emergency medical admission (OR $=0.68$, CI: 0.47-0.97).

The directly age-standardised mortality rates (ASR) per 10,000 person years were unchanged between study periods ([Period 1 ASR 299, CI: 251-348][Period 2 ASR 281, CI: 233-328]). 
On multivariate analysis, age, deprivation status, co-morbidity, and hospital length of stay were associated with increased 3-year mortality in both study periods.

Conclusion The overall mortality after hospitalisation for CD has not altered, although mortality associated with emergency medical admission has decreased, and now does not differ from rates after emergency surgical admission.

\section{REFERENCE}

1 Kennedy NA, Clark DN, Bauer J, et al. Nationwide linkage analysis in Scotland to assess mortality following hospital admission for Crohn's disease: 1998-2000. Aliment Pharmacol Ther 2012;35:142-53

Disclosure of Interest N. Ventham: None Declared, N. Kennedy: None Declared, A. Duffy: None Declared, D. Clark: None Declared, A. Crowe: None Declared, A. Knight: None Declared, J. Nicholls Grant/research support from: A grant was obtained from AbbVie Ltd to be administered by the North West London Hospital Trust (NWLHT) on behalf of Prof Nicholls, to allow funding of ISD and Corvus Communications for their work on the project. In the context of the work presented in this manuscript and in consideration of BMJ guidance, none of the authors have any competing or other conflict of interest, J. Satsangi: None Declared.

\section{PWE-085 AUDIT OF OUTCOMES OF A MANAGED 5 ASA SWITCHING PROGRAM}

${ }^{1} \mathrm{~N}$ Taylor*, ${ }^{2} \mathrm{D}$ Chan, ${ }^{3} \mathrm{~J}$ Bowey, ${ }^{1} \mathrm{~F}$ Cummings. 'Gastroenterology, University Hospital Southampton NHS Foundation Trust, Southampton, UK; ${ }^{2}$ Gastroenterology, Lymington New Forest Hospital, Lymington, UK; ${ }^{3}$ Medicines Management, Southampton City CCG, Southampton, UK

\subsection{6/gutjnl-2014-307263.345}

Introduction 5-aminosalicylic acid (5-ASA) preparations are used to induce and maintain remission in ulcerative colitis (UC). The cost of the recommended maintenance dose for oral 5ASA preparations varies from $86 p$ and 208p per day (MIMMS 2011). Switching to cheaper 5ASAs has been suggested as a possible drug cost saving. The BNF states that preparations are not interchangeable but with little direct evidence to support this statement. The aim of this pilot study is to gain preliminary data on a primary care based 5ASA switching programme.

Methods Salofalk granules (Dr Falk Pharma) and Pentasa (Ferring Pharmaceuticals) were identified as the cheapest 5ASA items prescribed in 31 GP practices. An initial pilot study in one practice revealed that only $5 / 21$ patients responded to a written invitation alone and successfully switched to Salofalk. We therefore developed a managed programme utilising a community based gastroenterology nurse specialist in 4 other practices.

A written invitation detailing the rationale for switching 5ASA was sent to all appropriate patients who were then phoned a week later. If patients agreed to the switch, the GP surgery was contacted by the Nurse Specialist to change the repeat prescription. Patients were switched to Salofalk in 2 practices and Pentasa in the other 2. GP and hospital records were then examined 6 months post-switch to assess for evidence of patient acceptability and tolerability.

Results 120 patients (56 male, 64 female) with a mean age of 50 years were identified as being on a 5-ASA preparation (oral or topical). 56 (47\%) were under either virtual or hospital gastroenterology follow-up. 64 patients with ulcerative colitis were taking oral 5-ASAs. 21 (33\%) were already taking Salofalk or Pentasa. Of the remaining 43 patients, 24 (56\%) agreed to the switch, 10 (23\%) declined, and 9 (21\%) did not respond to the invitation or telephone call. Of the 24 patients who agreed to switch, only 17 (71\%) completed the process. $15(88 \%)$ remained on the new 5-ASA for at least 6 months, with reasons for discontinuation cited as preference for the previous preparation or diarrhoeal symptoms.

Conclusion Conducting a managed 5ASA switching programme is feasible with 17/43 eligible patients successfully switched with $15 / 17$ continuing on these preparations. Areas for development include following up patients who initially agreed but failed to switch, recording more robustly any flares, involving secondary physicians and assessing adherence and cost savings.

This study provides preliminary evidence to develop a large scale study in this important area.

Disclosure of Interest N. Taylor: None Declared, D. Chan: None Declared, J. Bowey: None Declared, F. Cummings Speaker bureau with: Ferring Pharmaceuticals, Conflict with: Advisory board and educational support Ferring Pharmaceuticals. Educational support: Dr Falk, Warner Chilcott and Shire.

\section{PWE-086 DETERMINING STEM CELL AND CRYPT DYNAMICS IN INFLAMMATORY BOWEL DISEASE}

${ }^{1} \mathrm{~N}$ Jawad ${ }^{*},{ }^{1} \mathrm{~J}$ Crook, ${ }^{1} \mathrm{~A}-\mathrm{M}$ Baker, ${ }^{1} \mathrm{~B}$ Cereser, ${ }^{2} \mathrm{M}$ Rodriguez-Justo, ${ }^{1} \mathrm{~T}$ Graham, ${ }^{1} \mathrm{~N}$ Wright, 'S McDonald. 'Tumour Biology, Bart's Cancer Institute, London, UK; ${ }^{2}$ Histopathology, University College Hospital, London, UK

\subsection{6/gutjnl-2014-307263.346}

Introduction Inflammatory bowel disease (IBD) confers a high risk of development of colitis-associated colorectal cancer (CACRC) in patients with extensive colitis. It is believed that a field effect, resulting from chronic inflammation and clonal outgrowth is present in ulcerative colitis (UC) patients, and this promotes the accumulation of protumourigenic clones via increased crypt fission rates. Increased rates of crypt fission may explain the mass expansion of protumourigenic mutations across the whole length of the bowel in a very short time period as observed in patients with CACRC (Leedham et al., 2009; Galandiuk et al., 2012).

Methods Fresh frozen normal colon $(\mathrm{n}=15)$ and UC colon ( $\mathrm{n}$ =6) tissue samples were collected and sectioned in an en face orientation. Two-colour enzyme histochemistry for cytochrome $\mathrm{c}$ oxidase (CCO) activity was performed to identify clonal populations. Adjacent crypts completely deficient of CCO activity were recorded as a 'CCO deficient patch' and those with a fraction of the crypt that was CCO-deficient were designated as 'partial crypts'. The CCO-deficient clone fraction in partial crypts was estimated by recording the number of pixels within CCO-deficient (blue) and CCO-proficient (brown) areas. The number and size of clonal patches in colitis patients was compared to nonUC controls.

Results As in the healthy colon, CCO-deficient clonal patches accumulate in an age dependent fashion in the UC colon. The mean number of crypts within a CCO-deficient patch was statistically significantly larger $(p<0.05)$ in the UC colon than in the normal colon. In addition we observe a larger percentage of wholly and partially CCO-deficient crypts in the UC colon when compared to the normal controls.

Conclusion The proliferative drive induced by continuous inflammation and mucosal repair in UC appears to promote the expansion of CCO-deficient patches. The increase in the proportion of wholly and partially mutated crypts in UC could be explained by crypt hyperplasia with more stem cells present driving fission. This increased rate of clonal expansion may contribute to the 